\title{
NUMERICAL SIMULATION OF A HEAT TREATMENT PROCESS OF 7055-SiCP COMPOSITES USING COMPUTATIONAL FLUID DYNAMICS
}

\author{
Jing Su${ }^{1 *}$, Sunan Yuan ${ }^{1}$ \\ ${ }^{1}$ Henan Polytechnic Institute, Nanyang 473000, Henan, China. \\ *Corresponding author: Jing $\mathrm{Su}$ \\ Email: sujinghpi@yeah.net
}

\begin{abstract}
The purpose of this study is to explore the phase transformation and property change of 7055-SiCp composite in heat treatment process, and find out the method to improve the heat treatment process. With the help of the most commonly used commercial computational fluid dynamics (CFD) software in numerical simulation, a numerical simulation model describing the heat treatment process of 7055-SiCp composite is established. Moreover, the numerical simulation experiment of the change of the composite material in the heat treatment process is taken as the research object. CFD technology is used as a research tool. By means of numerical simulation and commercial software, a model describing the internal changes of heat treatment process of 7055$\mathrm{SiCp}$ aluminum matrix composites is established to analyze the internal structure transformation, structure transformation and physical properties changes. The results show that there is a second phase aluminum copper $\mathrm{Al} 2 \mathrm{Cu}$ in the composite matrix. In addition, the static recrystallization phenomenon and recrystallization structure appear during annealing and solution treatment of heat treatment process. Annealing at $300^{\circ} \mathrm{C}$ for $20 \mathrm{~h}$ can significantly increase the elongation of the material by about $76 \%$, but the tensile and compressive properties and yield strength of the material also decrease by $47 \%$ and $52 \%$, respectively. The numerical simulation software is used to discretize the macro phase transformation problem to further optimize the heat treatment process. Through the simulation of material heat treatment, the advantages and disadvantages of the process are analyzed accurately, to achieve accurate control and improvement of the process. It has a strong application value for the optimization of material performance after heat treatment, and has great reference significance for the follow-up research work.
\end{abstract}

Keywords: Computational Fluid Dynamics; Numerical Simulation; Composite Materials; Heat Treatment Process.

\section{Introduction}

Composite material [1] is a new material that people use advanced material preparation technology to optimize the composition of materials with different properties. It has excellent physical and mechanical properties, and can fill the performance weakness of each component, so as to achieve the comprehensive performance advantages that a single component material does not have. At present, it is widely used in aviation, automobile, communication and other industrial fields, and plays a vital role in the development of human society. In previous studies, silicon carbide particles (SiCp) are added into aluminum materials to make SiCp aluminum matrix composites [2]. Because of the physical characteristics of high strength, high modulus, high hardness, wear resistance and high temperature resistance of $\mathrm{SiC}$ materials, the shortcomings of low melting point and low hardness of the original aluminum materials are changed, so it become a research hotspot in many countries. At present, the research on the heat treatment of SiCp aluminum matrix composites is relatively less, and mainly relies on the traditional test methods, which cannot go deep into the interior of the material. Therefore, this exploration aims to use computational fluid dynamics (CFD) technology to simulate the heat treatment process of this material.

With the development of CFD after 1990s [3], the method of using computer simulation instead of manpower to solve complex mathematical equations has been popularized. CFD is used as a research tool to simulate the two-dimensional and threedimensional material flow in the real world. At present, CFD technology has been widely used in China and foreign countries, so CFD is used to detect the internal complex fluid mechanics properties in 
order to make appropriate optimization. Present commercial software such as STAR-CD, CFX, Phenics and fluent are generally used in CFD research. CFD software can broaden the scope of experimental research and reduce the cost and workload. It can generally produce a variety of optimized physical models, and there are suitable numerical solutions for the flow characteristics of each physical problem $[4,5]$.

Numerical simulation about the heat treatment process of 7055-SiCp composites is performed based on CFD. The relevant models are established. The model and model parameters are studied comprehensively and systematically. The CFD numerical simulation method for heat treatment process of 7055-SiCp composites is established. The CFD numerical simulation method is applied to the simulation calculation of heat treatment process performance of composite materials, and the physical and mechanical properties of materials in the process are analyzed, which provides theoretical guidance for the subsequent theoretical research.

\section{Methodology}

\subsection{Theoretical Basis of CFD}

All fluid flows follow the following three laws: the law of conservation of mass; the law of conservation of momentum (Newton's second law); and the law of conservation of energy, which can be described by some mathematical relations, in order of continuity equation, momentum equation, and energy equation. CFD is to use the discrete algebraic form to replace the integral or differential in the above equation, and solve it, so as to get the time and space distribution of the flow parameters.

\section{(1) Continuity equation}

In the process of fluid flow, the sum of the net mass of fluid flowing in and out of the control body per unit time should be equal to the mass increased or decreased due to the change of internal density [6], as shown in Figure 1.

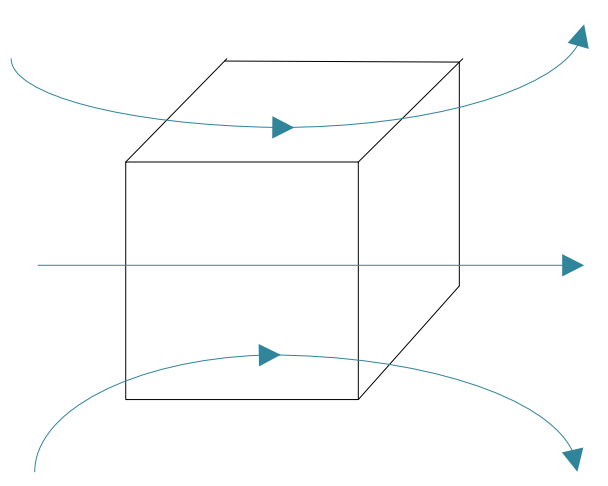

Figure 1: Corresponding diagram of different forms of continuity equation
By characterizing the fluid of control body as infinitesimal micro cluster with fixed spatial position, the conservative differential form can be obtained; by characterizing the fluid of control body as the infinitesimal micro cluster with constant mass of fluid motion, the non-conservative differential form can be obtained [7]. (1) is non-conservative equation and (2) is conservation equation.

$$
\begin{aligned}
& \frac{D \rho}{D \mathrm{t}}+\rho \nabla \cdot V=0 \\
& \frac{D \rho}{D \mathrm{t}}+\nabla \cdot(\rho V)=0
\end{aligned}
$$

Material derivative: $\frac{D \rho}{D \theta}$ represents the observer's motion in the fluid at exactly the same velocity as the fluid velocity. At this time,

$$
\boldsymbol{u}_{\mathrm{x}}=\frac{d x}{d \theta} \quad \boldsymbol{u}_{y}=\frac{d y}{d \theta} \quad \boldsymbol{u}_{z}=\frac{d z}{d \theta}
$$

$u$ is the velocity vector at a certain point at time $t$, and $u=\left\{u_{x}, u_{y}, u_{z}\right\} ; u_{x}$ is the derivative of displacement of fluid mass point in the direction of coordinate axis $x$ with time; $u_{y}$ is the derivative of displacement in the direction of $y$ with time; $u_{z}$ is the derivative of displacement in the direction of $z$ with time. $\rho$ is the density of the fluid, $\nabla$ is the Laplacian operator, and $V$ is the element of volume.

$$
\frac{D \rho}{D t}=\frac{\partial \rho}{\partial t}+\boldsymbol{u}_{x} \frac{\partial \rho}{\partial x}+\boldsymbol{u}_{y} \frac{\partial \rho}{\partial y}+\boldsymbol{u}_{z} \frac{\partial \rho}{\partial z}
$$

\section{(2) Momentum equation}

Infinitesimal microcapsule is studied, its force is equal to the product of mass and acceleration, and its force includes gravity, body force, pressure and surface force. The body force is caused by electromagnetic force, and the surface force is caused by viscous force, as shown in Figure $2[8,9]$.

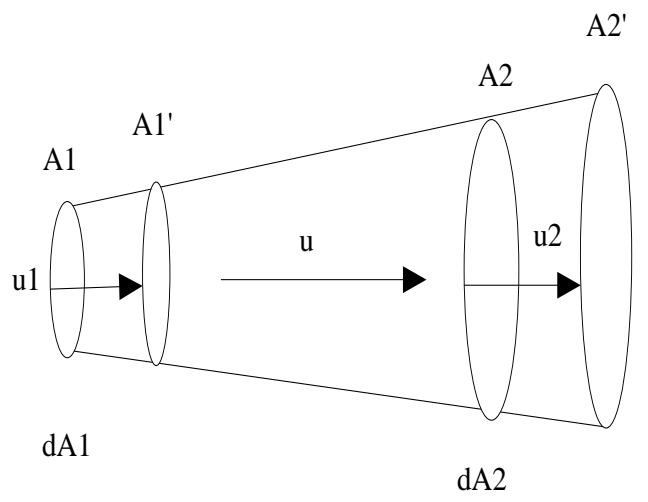

Figure 2: Schematic diagram of momentum equation 
The following equations (5)-(14) are used for calculation:

Non-conservative equation:

$$
\begin{aligned}
& \rho \frac{D u}{D t}=-\frac{\partial p}{\partial x}+\frac{\partial \tau_{x x}}{\partial x}+\frac{\partial \tau_{y x}}{\partial y}+\frac{\partial \tau_{z x}}{\partial z}+\rho f_{x} \\
& \rho \frac{D v}{D t}=-\frac{\partial p}{\partial y}+\frac{\partial \tau_{x y}}{\partial x}+\frac{\partial \tau_{y y}}{\partial y}+\frac{\partial \tau_{z y}}{\partial z}+\rho f_{y} \\
& \rho \frac{D w}{D t}=-\frac{\partial p}{\partial z}+\frac{\partial \tau_{x z}}{\partial x}+\frac{\partial \tau_{y z}}{\partial y}+\frac{\partial \tau_{z z}}{\partial z}+\rho f_{z}
\end{aligned}
$$

The unit mass force is a vector force with direction and magnitude. The equation is as follows:

$$
\vec{f}=f_{x} \vec{i}+f_{y} \vec{j}+f_{z} \vec{k}
$$

$f_{x}$ is the unit mass force in $x$ direction; $f_{y}$ is the unit mass force in $y$ direction; $f_{z}$ is the unit mass force in $z$ direction. $P$ is the pressure at the mass point, $u$ is the velocity in the $x$ direction, $v$ is the velocity in the $y$ direction, $w$ is the velocity in the $z$ direction, $\tau$ represents the shear stress on the edge of the element, and $\rho$ is the fluid density.

$$
\begin{aligned}
& \frac{D \rho}{D t}=\frac{\partial \rho}{\partial t}+\boldsymbol{u}_{x} \frac{\partial \rho}{\partial x}+\boldsymbol{u}_{y} \frac{\partial \rho}{\partial y}+\boldsymbol{u}_{z} \frac{\partial \rho}{\partial z} \\
& \frac{D w}{D t}=\frac{\partial w}{\partial t}+\boldsymbol{u}_{x} \frac{\partial w}{\partial x}+\boldsymbol{u}_{y} \frac{\partial w}{\partial y}+\boldsymbol{u}_{z} \frac{\partial w}{\partial z} \\
& \frac{D v}{D t}=\frac{\partial v}{\partial t}+\boldsymbol{u}_{x} \frac{\partial v}{\partial x}+\boldsymbol{u}_{y} \frac{\partial v}{\partial y}+\boldsymbol{u}_{z} \frac{\partial v}{\partial z}
\end{aligned}
$$

Conservation equation:

$$
\begin{aligned}
& \frac{\partial(\rho u)}{\partial t}+\nabla \cdot(\rho u V)=-\frac{\partial p}{\partial x}+\frac{\partial \tau_{x x}}{\partial x}+\frac{\partial \tau_{y x}}{\partial y}+\frac{\partial \tau_{z x}}{\partial z}+\rho f_{x} \\
& \frac{\partial(\rho v)}{\partial t}+\nabla \cdot(\rho v V)=-\frac{\partial p}{\partial y}+\frac{\partial \tau_{x y}}{\partial x}+\frac{\partial \tau_{y y}}{\partial y}+\frac{\partial \tau_{z y}}{\partial z}+\rho f_{y} \\
& \frac{\partial(\rho w)}{\partial t}+\nabla \cdot(\rho w V)=-\frac{\partial p}{\partial y}+\frac{\partial \tau_{x z}}{\partial x}+\frac{\partial \tau_{x z}}{\partial y}+\frac{\partial \tau_{z z}}{\partial z}+\rho f_{z}
\end{aligned}
$$

$f_{x}$ is the unit mass force in $x$ direction; $f_{y}$ is the unit mass force in $y$ direction; $f_{z}$ is the unit mass force in $z$ direction.

\section{(3) Energy equation}

Energy equation refers to the equation reflecting the law of conservation of energy including internal energy when density, temperature and internal energy change.
The energy equation includes kinetic energy, potential energy and work (work done by pressure P or viscous force) of body force (such as gravity), and represents the mutual transformation of mechanical energy and internal energy in the fluid in the control body. The energy equation is shown in equation (15) and equation (16) $[10,11]$.

Non-conservative equation:

$$
\begin{aligned}
& \rho \frac{D}{D t}\left(e+\frac{\mathbf{V}^{2}}{2}\right) \\
& =\rho q+\frac{\partial}{\partial x}\left(k \frac{\partial T}{\partial x}\right)+\frac{\partial}{\partial y}\left(k \frac{\partial T}{\partial y}\right)+ \\
& \frac{\partial}{\partial z}\left(k \frac{\partial T}{\partial y}\right)-\frac{\partial(u p)}{\partial x}-\frac{\partial(v p)}{\partial y}- \\
& \frac{\partial(w p)}{\partial z}+\frac{\partial\left(u \tau_{x x}\right)}{\partial x}+\frac{\partial\left(u \tau_{x y}\right)}{\partial y}+\frac{\partial\left(u \tau_{z x}\right)}{\partial z} \\
& +\frac{\partial\left(v \tau_{x y}\right)}{\partial x}+\frac{\partial\left(v_{\tau_{y y}}\right)}{\partial y}+\frac{\partial\left(v \tau_{z y}\right)}{\partial z}+ \\
& \frac{\partial\left(w \tau_{x z}\right)}{\partial x}+\frac{\partial\left(v \tau_{y z}\right)}{\partial y}+\frac{\partial\left(v \tau_{z z}\right)}{\partial z}+\rho f \cdot V
\end{aligned}
$$

Conservation equation:

$$
\begin{aligned}
& \frac{\partial}{\partial t}\left[\rho\left(\mathrm{e}+\frac{V^{2}}{2}\right)\right]+\nabla \cdot\left[\rho\left(\mathrm{e}+\frac{V^{2}}{2}\right) V\right] \\
& =\rho q+\frac{\partial}{\partial x}\left(k \frac{\partial T}{\partial x}\right)+\frac{\partial}{\partial y}\left(k \frac{\partial T}{\partial y}\right)+\frac{\partial}{\partial z}\left(k \frac{\partial T}{\partial y}\right) \\
& -\frac{\partial(u p)}{\partial x}-\frac{\partial(v p)}{\partial y}-\frac{\partial(w p)}{\partial z}+\frac{\partial\left(u \tau_{x x}\right)}{\partial x} \\
& +\frac{\partial\left(u \tau_{x y}\right)}{\partial y}+\frac{\partial\left(u \tau_{z x}\right)}{\partial z}+\frac{\partial\left(v \tau_{x y}\right)}{\partial x}+\frac{\partial\left(v \tau_{y y}\right)}{\partial y} \\
& +\frac{\partial\left(v \tau_{z y}\right)}{\partial z}+\frac{\partial\left(w \tau_{x z}\right)}{\partial x}+\frac{\partial\left(v \tau_{y z}\right)}{\partial y}+\frac{\partial\left(v \tau_{z z}\right)}{\partial z} \\
& +\rho f \cdot V
\end{aligned}
$$

$\ell$ is the internal energy of the liquid, $T$ is the temperature of the liquid, $q$ is the unit heat of the liquid, $k$ is the energy coefficient of the liquid, $u$ is the velocity in $x$ direction at time $t, v$ is the velocity in $y$ direction, and $w$ is the velocity in $z$ direction.

$f_{x}$ is the unit mass force in $x$ direction; $f_{y}$ is the unit mass force in $y$ direction; $f_{z}$ is the unit mass force in $z$ direction. $\nabla$ is the Laplace operator. 


\subsection{Overview of Fluid Model}

\section{(1) Turbulence model}

Fluid flow can be divided into laminar flow and turbulent flow. Laminar flow is a flow state of fluid. When the fluid flows in the pipe, its mass point moves in a smooth straight line along the direction parallel to the pipe axis. This kind of flow is called laminar flow or stagnant flow, or linear flow. The velocity of fluid is the largest in the center of the pipe and the smallest near the wall. The ratio of average velocity to maximum velocity is equal to 0.5 . According to the Reynolds experiment, when the Reynolds number $\operatorname{Re}<2320$, the flow state of the fluid is laminar; turbulence is another flow state of the fluid. When the flow velocity is very small, the fluid flows in layers and does not mix with each other, which is called laminar flow, also known as steady flow or sheet flow; when the flow velocity increases gradually, the streamline of the fluid begins to appear wavy oscillation, and the frequency and amplitude of oscillation increase with the increase of the flow velocity, which is called transient flow; when the flow velocity increases to a large value, the streamline is no longer clear, and there are many small vortices in the flow field. The laminar flow is destroyed and there is not only sliding but also mixing between adjacent layers. At this time, the fluid moves irregularly, and there is a partial velocity perpendicular to the axis of the flow tube. This kind of motion is called turbulence. Figure 3 shows the difference [12].

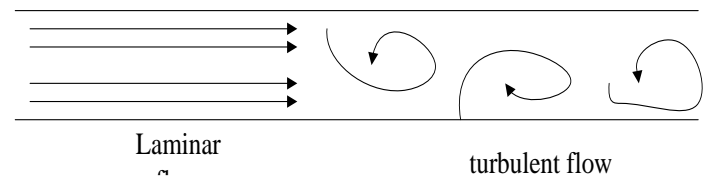

Figure 3: Laminar and turbulent flow

It is generally characterized by Reynolds number (Re). When $\operatorname{Re} \leq 2000$, it is laminar flow, and when Re $\geq 2000$, it is turbulent flow. The action mechanism and magnitude of viscous force and inertial force are different under different flow conditions. The fluid layers interfere with each other and can transfer mass and momentum. The calculation equation is (17).

$$
R \mathrm{e}=\frac{\rho V d}{\mu}
$$

The traditional research methods cannot understand the turbulent flow in the fluidized bed, but the numerical simulation method can be used to analyze the problem from the perspective of discretization, which is more operable than the former. Fluent software tool provides a variety of turbulence models [13], and Table 1 shows its name and scope of application.
Table 1 Turbulence models and its application

\begin{tabular}{|l|l|}
\hline $\begin{array}{l}\text { Model } \\
\text { category }\end{array}$ & Application scope \\
\hline S-A & $\begin{array}{l}\text { Simulation of the internal or } \\
\text { external flow with general } \\
\text { complexity, or the boundary } \\
\text { layer flow in the pressure } \\
\text { gradient }\end{array}$ \\
\hline Standard & $\begin{array}{l}\text { Initial iteration, design selection } \\
\text { and parameter research }\end{array}$ \\
\hline RNG & $\begin{array}{l}\text { Design of the complex shear flow } \\
\text { with fast strain, medium vortex } \\
\text { and local turbulence }\end{array}$ \\
\hline Realizable & $\begin{array}{l}\text { Prediction of rotational flow } \\
\text { with general strength }\end{array}$ \\
\hline Standard & $\begin{array}{l}\text { the situation with reverse } \\
\text { pressure gradient }\end{array}$ \\
\hline SST & $\begin{array}{l}\text { It cannot be used to simulate } \\
\text { free shear flow }\end{array}$ \\
\hline RSM & $\begin{array}{l}\text { Simulation of complex three- } \\
\text { dimensional flows such as strong } \\
\text { vortex and rotational flow }\end{array}$ \\
\hline LES & $\begin{array}{l}\text { Simulation of transient large- } \\
\text { scale vortices }\end{array}$ \\
\hline eddy & $\begin{array}{l}\text { It improves the near wall } \\
\text { treatment of large eddy } \\
\text { simulation and is more practical } \\
\text { than LES model }\end{array}$ \\
\hline
\end{tabular}

$\mathrm{k}-\varepsilon$ model is divided into Standard model, RNG model and Realizable model; $\mathrm{k}-\omega$ model is divided into Standard model and SST model.

\section{(2) Multiphase flow model}

At present, the widely used numerical methods for multiphase flow are Euler-Euler method and Eular-Langrange method. The former is used to analyze the fluid motion of infinitesimal clusters (Euler), and the latter is used to analyze infinitesimal clusters (Lagrange method) [14].

Discrete phase model (DPM) is the computational model of Eular-Langrange method, which mainly focuses on the trajectory of discrete phase and requires discrete phase $\mathrm{V} \% \leq 10 \%$.

The models provided by Fluent software include Volume of Fluid (VOF) model, Mixture model, and Eulerian model. (1) VOF model: it is very sensitive to steady-state calculation, and is generally used to solve transient problems. Therefore, VOF model is suitable for simulating transient fluid flow with phase mixing and immiscibility. (2) Mixture model: the Mixture model is suitable for the exploration of interface law and the wide distribution of discrete phase. (3) Eulerain model: Eulerain model can simulate multiphase flow and interphase interaction, such as bubble column, floating, particle suspension and other complex problems with obvious interphase force [15]. 


\subsection{Heat Treatment Process of Composite Materials}

\section{(1) Composition of composite materials}

In 7055-SiCp composite matrix alloy, $\mathrm{SiC}$ particles are used as reinforcement to enhance its properties, and 7055 aluminum is used matrix. The specific composition of 7055 aluminum is as follows: the mass fraction of $\mathrm{Cr}$ is less than $0.04 \%$, the mass fraction of $\mathrm{Cu}$ is $2.0 \%$ to $2.6 \%$, the mass fraction of $\mathrm{Fe}$ is less than $0.15 \%$, the mass fraction of $\mathrm{Mg}$ is less than $1.8 \%$ to $2.3 \%$, the mass fraction of $\mathrm{Si}$ is less than $0.10 \%$, the mass fraction of $\mathrm{Ti}$ is less than $0.06 \%$, the mass fraction of $\mathrm{Zn}$ is between $7.6 \%$ and $8.4 \%$, the mass fraction of $\mathrm{Zr}$ is between $0.05 \%$ and 0.25 , and $\mathrm{Al}$ is the rest main body. The $\mathrm{SiC}$ used is 800 mesh. Figure 4 is a schematic diagram of the composite structure.
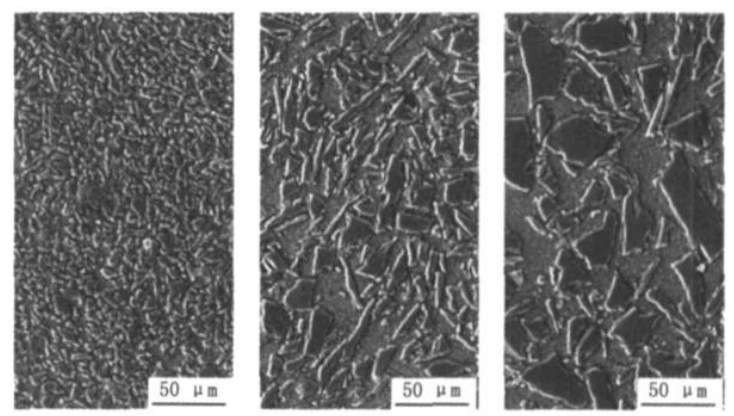

Figure. 4 Microstructure of 7055-SiCp composite matrix alloy

\section{(2) Quenching heat treatment process}

Quenching process $(\mathrm{QP})$ is a heat treatment process in which the steel is heated to the temperature above the critical temperature Ac3 (hypo eutectoid steel) or Ac1 (hypereutectoid steel) for a period of time to make it fully or partially austenitized, and then rapidly cooled to below Ms (or isothermal near MS) at a cooling rate higher than the critical cooling rate for martensite (or bainite) transformation. Generally, the solution treatment of aluminum alloy, copper alloy, titanium alloy, tempered glass and other materials or the heat treatment process with rapid cooling process is also called quenching. The purpose of quenching is to transform the undercooled austenite into martensite or bainite to obtain martensite or bainite structure, and then temper at different temperatures to greatly improve the rigidity, hardness, wear resistance, fatigue strength and toughness of the steel, so as to meet the different requirements of various mechanical parts and tools. The special physical and chemical properties such as ferromagnetism and corrosion resistance of some special steels can also be satisfied by quenching. The metal heat treatment process is that the metal workpiece is heated to a certain appropriate temperature and kept for a period of time, and then immersed in the quenching medium for rapid cooling. Commonly used quenching media are salt solution and water, mineral oil, and air [16]. Quenching can improve the hardness and wear resistance of metal workpieces, so it is widely used in various tools, molds, measuring tools and parts requiring surface wear resistance (such as gears, rolls, and carburized parts). Through the combination of quenching and tempering at different temperatures, the strength, toughness and fatigue strength of metal can be greatly improved, and the combination of these properties (comprehensive mechanical properties) can be obtained to meet different requirements.

In addition, quenching can also make some special properties of steel obtain certain physical and chemical properties, such as quenching can make permanent magnetic steel enhance its ferromagnetism, and make stainless steel improve its corrosion resistance. Quenching process is mainly used for steel parts. When the common steel is heated above the critical temperature, all or most of the original structure at room temperature will be transformed into austenite. Subsequently, the steel is immersed in water or oil for rapid cooling, and austenite transforms into martensite. Compared with other structures in the steel, martensite has the highest hardness.

The rapid cooling during quenching will cause internal stress in the workpiece. When it reaches a certain extent, the workpiece will be distorted or even cracked. Therefore, it is necessary to choose a suitable cooling method. According to the cooling method, the quenching process can be divided into four types: single liquid quenching, interrupted quenching, martempering and bainite isothermal quenching $[17,18]$.

\subsection{Setting of System Simulation Parameters}

\section{(1) Research objects}

The CFD software Fluent 6.3.26 is used for twodimensional numerical simulation. First, the CFD simulation method and platform of 7055-SiCp composite heat treatment process are established.

\section{(2) Modeling}

Before CFD simulation, it is necessary to establish the physical model of the research object. This step is carried out by the preprocessing software of Fluent software. Two-dimensional simulation is adopted. First, in the preprocessing software, the twodimensional geometric model is established, and then the effective area is calculated.

The model is divided into six kinds of grids with sizes of $1 \mathrm{~mm}, 2 \mathrm{~mm}, 3 \mathrm{~mm}, 4 \mathrm{~mm}, 5 \mathrm{~mm}$ and $6 \mathrm{~mm}$, and the number of grids is 221909, 54804, 24498, 13611, 8604 and 5979, respectively. Under the same operation conditions, the simulation calculation is carried out to find the optimal number of grids that meet the calculation accuracy and cost. 


\section{(3) Calculation methods and conditions}

Fluent 6.3.26 software is used to solve the problem. The separation solver is selected and the format is implicit. Two-dimensional(2D) simulation is selected and the time format is set to unsteady. The standard $\mathrm{k}-\varepsilon$ model is selected for multiphase flow model.

Physical condition setting: the operating pressure is set at $101.325 \mathrm{kpa}$, and the gravity acceleration is set vertically downward.

The semi-implicit algorithm for pressure coupled equations is used to solve Navier-Stokes equations. The First Order Upwind scheme is used to discretize the beam, volume fraction, turbulent kinetic energy and turbulent dissipation rate, and the default relaxation factor is used. The simulation process is unsteady, and the steps of $0.001 \mathrm{~s}, 0.003 \mathrm{~s}$ and $0.005 \mathrm{~s}$ are used for iteration. Each time step is iterated 50 times, and the convergence of each parameter is set to 0.001 [19].

A simulation example is used to illustrate the application of dynamic fuzzy network in nonlinear system identification. The identification object is shown in equation (18).

$$
y(\mathrm{t}+1)=\frac{y(\mathrm{t}) \mathrm{y}(\mathrm{t}+1)[\mathrm{y}(\mathrm{t})+2.5]}{1+y^{2}(\mathrm{t})+\mathrm{y}^{2}(\mathrm{t}-1)}+b(\mathrm{t})
$$

Equation (18) suggests that the input variables of the system are $y(\mathrm{t}), \mathrm{y}(\mathrm{t}+1)$, and $b(\mathrm{t})$, and the output variable is $\mathrm{y}(\mathrm{t}-1)$. According to the output of the system, the object can be written in the form of equation (19) [20, 21].

$$
\hat{y}(t-1)=f(y(t), \mathrm{y}(t+1), \mathrm{b}(t))
$$

The input of the system is as follows: $u(\mathrm{t})=\sin (2 \pi t / 20), \mathrm{y}(0)=0, \mathrm{y}(1)=0.30$ sets of data samples are selected as the dataset. Among them, 20 groups of data are used to train the network and generate the corresponding model, and 10 groups of data are used to verify the generated model. The initial values of network parameters are set as $k_{d \max }=6, k_{d \text { min }}=0.4, \gamma=0.98, \beta=0.9$, width $=2, \quad e_{\max }=1, \quad e_{\text {min }}=0.3, \quad k=1.1$, $k_{w}=1.1$ and $k_{\text {err }}=0.002$, respectively.

\section{Results and Discussion \\ 3.1 Distribution of Internal Quenching Residual Stress}

Figure 5 shows the research path of 7055-SiCp aluminum matrix composite plate. Figure 6 shows the three-dimensional stress distribution of quenching residual stress in Xc, Yc and Zc paths of 7055-SiCp aluminum matrix composite plate.

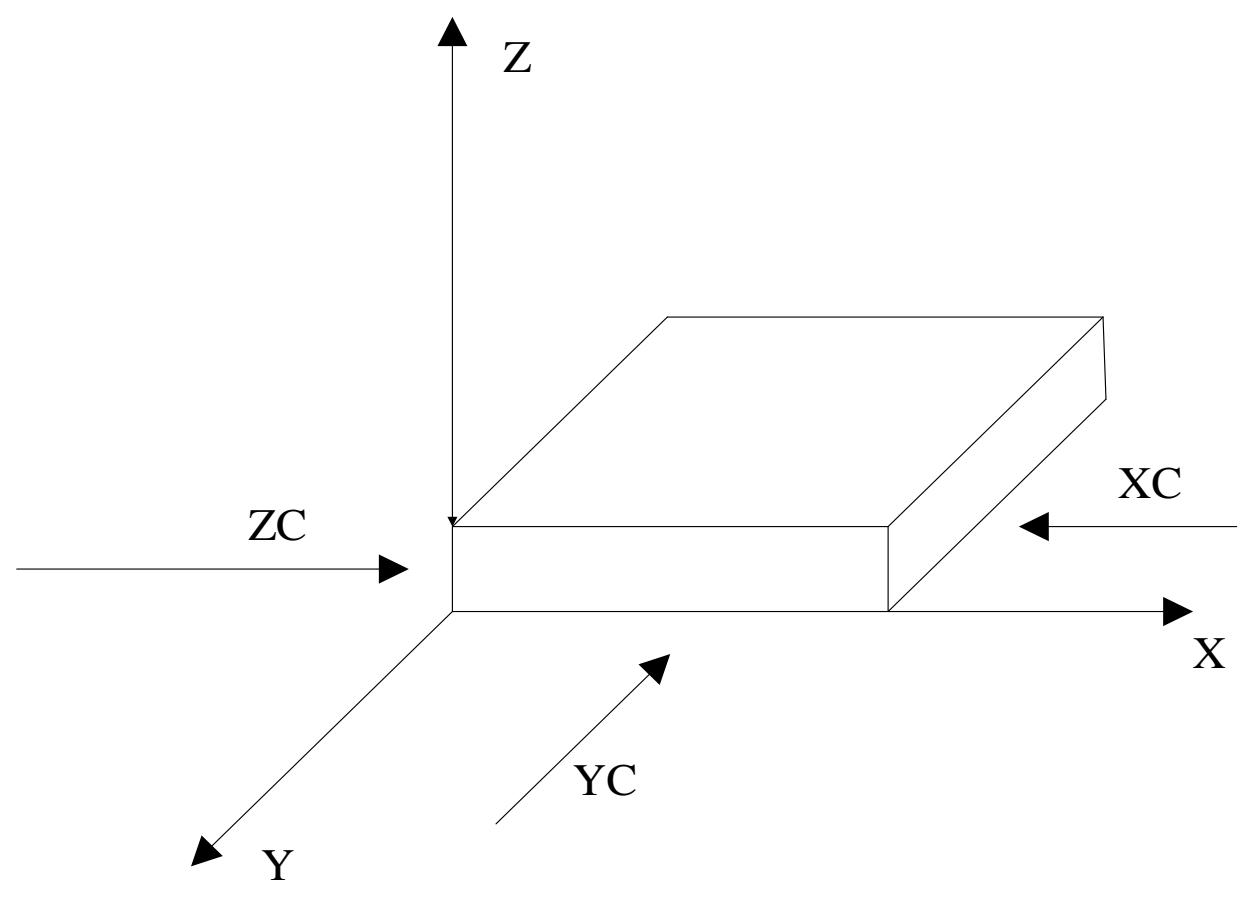

Figure 5: Schematic diagram of research path 

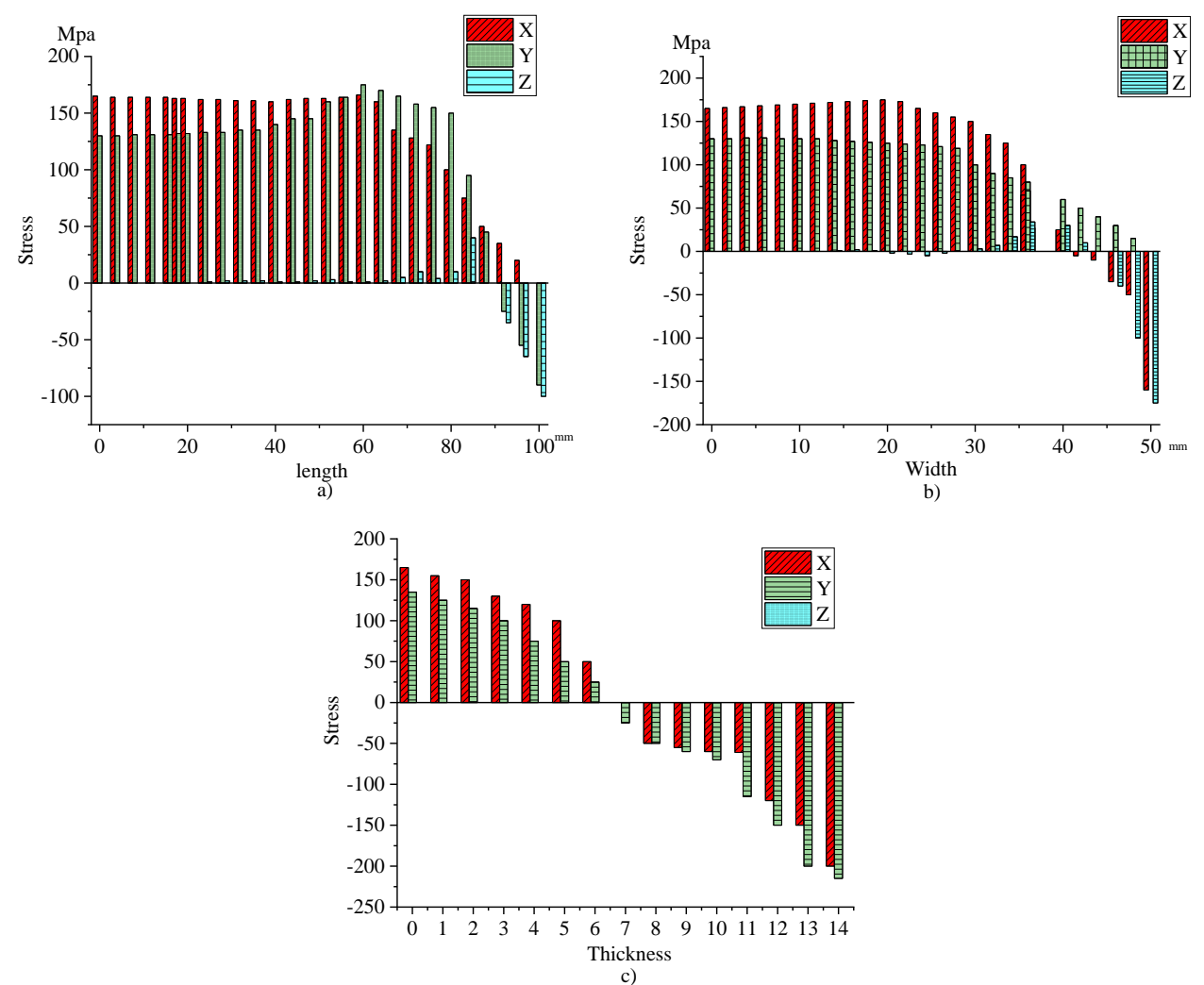

Figure 6: Distribution of quenching residual stress of 7055 aluminum alloy thick plate: $a: X c$ path; b: Yc path; c: Zc path

Figure 6 reveals the distribution characteristics of quenching residual stress: the X-direction quenching residual stress on $\mathrm{Z}$ path is $-201.8 \mathrm{Mpa}$ to $175.7 \mathrm{Mpa}$, and the Y-direction quenching residual stress is $-179.4 \mathrm{Mpa}$ to $134.8 \mathrm{Mpa}$. The stress distribution in the two directions is similar, which is in the form of external pressure and internal tension. The surface bears compressive stress, while the internal bears tensile stress. The Z-direction stress in the middle region of the thick plate is very small, almost zero, which can be ignored. Therefore, the quenching residual stress of the thick plate can be simplified as plane stress state. The maximum stress of Xc path is at one eighth of the internal stress, and the maximum value of Yc path is at one half of the internal stress.
The results show that the distribution of residual stress is anisotropic; the closer it is to the edge, the smaller the value of residual stress is, because the edge zone can be regarded as $2 \mathrm{D}$ heat transfer at the beginning of quenching; the cooling speed of edge zone is faster than that of the inside of plate, and the quenching strength of edge zone is higher during quenching, so the similar stress decreases.

\subsection{Distribution of Residual Stress during Surface Quenching}

Figure 7 shows the distribution of quenching residual stress of 7055 aluminum plate in Xs and Ys directions.
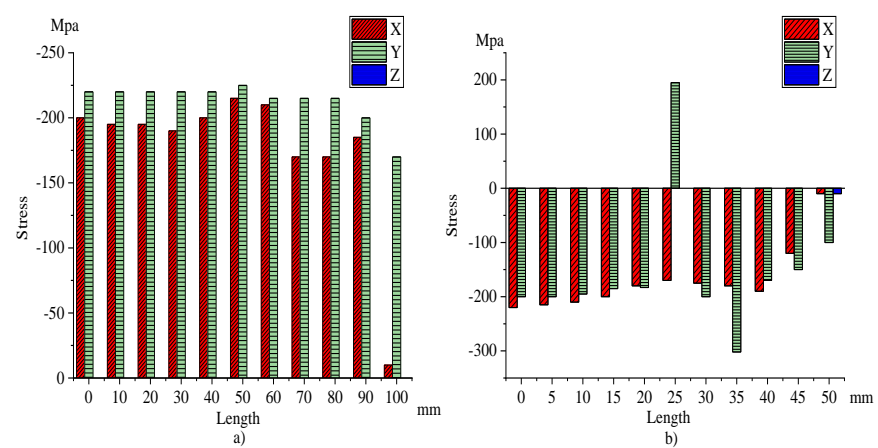

Figure 7: Distribution of quenching residual stress of 7055 aluminum plate in Xs and Ys directions a) Xs direction b) Ys direction 
Figure 7 is the simulated distribution diagram of quenching residual stress data of $7055-\mathrm{SiCp}$ aluminum alloy composite in Xs and Ys directions.

The surface heat treatment residual stress of composite plate is surface compressive stress. Moreover, the residual stress in the fixed $\mathrm{Z}$ direction is very small and can be ignored, so the stress distribution of composite plate can be regarded as a 2D plane state.

The change of $\mathrm{X}$-direction residual stress in $\mathrm{Xs}$ direction is less than $20 \%$ in the range of $0-100 \mathrm{~mm}$, and the change of Y-direction residual stress in Xs direction is less than $5 \%$ in the range of $0-100 \mathrm{~mm}$; the change of Y-direction residual stress in $\mathrm{Xs}$ direction is less than $20 \%$ in the range of $0-30 \mathrm{~mm}$, and the change of Y-direction residual stress in Ys direction is very small in the range of $0-30 \mathrm{~mm}$, which can be ignored, and the change area of Ys direction residual stress is about 30\% larger than that of Xs direction.

\subsection{Comparison of Simulation Results and Experimental Results}

Figure 8 shows the comparison between the experimental results and the simulation results of the quenching residual stress of 7055-SiCp composite in the thickness direction.

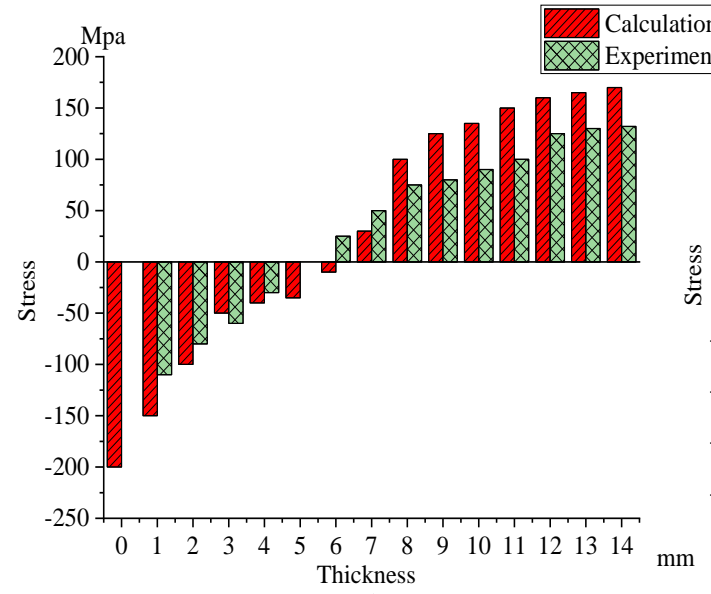

a)

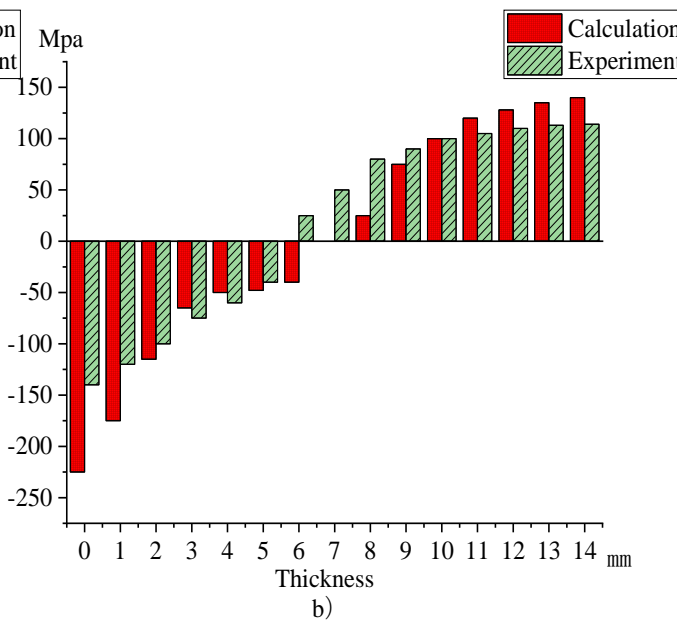

Figure 8: Experimental results and simulation results of quenching residual stress a) stress in $X$ direction b) stress in Y direction

In the Xs direction, four position points are equidistant tested. The results show that the average rolling stress of surface quenching residual stress is $172.6 \mathrm{MPa}$, and the fluctuation is less than $15 \%$. The predicted surface quenching residual stress is basically unchanged, and the value is $-185 \mathrm{MPa}$. The average value of transverse stress of surface quenching residual stress measured is $-220 \mathrm{MPa}$, and the fluctuation is less than $10 \%$. The predicted surface quenching residual stress is basically unchanged, and the value is $-230 \mathrm{MPa}$. The error may be due to the difference between the simulation results and the surface quenching residual stress caused by the uneven surface heat transfer. The measured results are basically consistent with the predicted results, which indicates that the simulation model can accurately predict the distribution of surface quenching residual stress.

(1) For 7055-SiCp composite plate, the surface quenching residual stress is compressive stress, and the core quenching residual stress is tensile stress. The maximum of the surface residual compressive stress is near the boundary, and the maximum of the central residual tensile stress is in the center.
In the middle region of thick plate, the Z-direction stress is almost zero, which can be ignored. The distribution of quenching residual stress can be approximately plane stress distribution.

(2) According to the comparison between the measured and simulated results of the surface quenching residual stress, the measured results are basically consistent with the predicted results, and the simulated and measured values of the thickness direction quenching residual stress are also in good agreement with the distribution trend. It shows that the simulation can accurately predict the distribution of 7055-SiCp composite plate.

(3) The formation process of quenching residual stress is as follows. At the initial stage of cooling, the core is in the state of compressive stress, and the surface is in the state of tensile stress. With the prolongation of quenching time, at about $2 \mathrm{~s}$, the core begins to change into tensile stress state, and the surface becomes compressive stress state. After cooling, the tensile stress state of the center and the compressive stress state of the surface are formed. 


\section{Conclusion}

The finite element model of stress distribution in quenching process of 77055-SiCp composite plate is established, and the quenching boundary conditions are established. Through the actual measurement, the feasibility of numerical simulation is verified, and the distribution law of quenching stress is calculated.

The simulation results are compared with the actual results, and the distribution of quenching residual stress is analyzed. Therefore, the numerical simulation can correctly simulate the heat treatment process of 77055-SiCp composite, which is helpful for the future research on the internal change model of heat treatment process of 7055-SiCp composite, and the analysis of internal structure transformation, structure transformation and physical properties change.

Although the original research goal has been basically achieved and some valuable research conclusions have been obtained, due to limited academic literacy, there are still many deficiencies in the research process. The research conclusions may be limited by the following three factors. (1) The compatibility of numerical simulation model is insufficient. (2) Lots of actual measurement data cannot be obtained for comparison with numerical simulation, which also is the direction for future research.

The future research will focus on: (1) further improving the compatibility of the model to make it more realistic; (2) further understanding the actual situation so as to obtain a lot of measured data.

\section{References}

[1] Maglio S., Felipe V., Rodrigues M. (2019) Benefits and Risks in the Use of Composite Materials in Solar Vehicles. Key Engineering Materials, 2(754), 51-54.

[2] Mousavian R., Khosroshahi R., Yazdani S. (2018) Fabrication of aluminum matrix composites reinforced with nano- to micrometer-sized $\mathrm{SiC}$ particles. Materials and Design, 5(2), 54-56.

[3] Kolehmainen J., Elfvengren J., Saarenrinne P. (2018) A measurement-integrated solution for particle image velocimetryand volume fraction measurements in a fluidized bed. International Journal of Multiphase Flow, 1(56), 72-80.

[4] Nouman A. (2018) Mesoscale Modeling and CFD Simulation of Gas-fluidized Bed with Binary Particle Mixture.University of Chinese Academy of Sciences: Institute of Process Engineering, 5(22), 22-35.

[5] Moldavanov A. Randomized Continuity Equation Model of Energy Transport in Open System. (2017) International Conference on Applied Mathematics, Simulation and Modellin, 2(5), 232251.

[6] Kevin R., Dominic W. (2021) 3D flow simulation of a baffled stirred tank for an assessment of geometry simplifications and a scale-adaptive turbulence model. Chemical. Engineering Science, 5(21), 231.

[7] Rajaratnam N. (2018) Horizontal Injection of GasLiquid Mixtures in a Water Tank. Journal of Hydraulic Engineering, 134(12), 1722-1731.

[8] Sugiyama K., Takaigi S., Matsumoto Y. (2017) Multi-scale analysis of bubbly flows. Computer Methods in Applied Mechanicsa \& Engineering, 191(6-7), 689-704.

[9] Klasik A., Maj M., Pietrzak K., et al. (2018) Fatigue Life and Microstructure after Multiple Remelting of A359 Matrix Composites Reinforced with SiC Particles. Archives of Metallurgy \& Materials, 61(4), 101-115.

[10] Ranaivoson T., Andriambololona R., Rakotoson H. (2017) Eigenvalue equation for momentum dispersion operator and properties of phase space wavefunctions, 3(22), 98-125.

[11] Auclair J., Lemieux J., Tremblay L. (2017) Implementation of Newton's method with an analytical Jacobian to solve the $1 \mathrm{D}$ sea ice momentum equation. Journal of Computational Physics, 3(56), 192-213.

[12] Jadli U., Mohd-Yasin F., Moghadam H. (2020) A Simple Equation for the Energy Stored by Voltage-Dependent Capacitances. Transactions on Power Electronics, 1(99), 1-2.

[13] Babaniyi O., Oberai A., Barbone P E. (2017) Recovering vector displacement estimates in quasistatic elastography using sparse relaxation of the momentum equation. Materials and Design, 25(3), 103-155.

[14] Guissart A., Bernal L., Dimitriadis G. (2017) PIVbased estimation of unsteady loads on a flat plate at high angle of attack using momentum equation approaches. Experiments in Fluids, 58(5), 53-54.

[15] Epple P., Steppert M., Wunder L. (2017) Verification of Torricelli's Efflux Equation with the Analytical Momentum Equation and with Numerical CFD Computations. Applied Mechanics and Materials, 2(6), 871-901. 
[16] Simpson C., Grand R. (2017) Quenching and ram pressure stripping of simulated Milky Way satellite galaxies. Monthly Notices of the Royal Astronomical Society, 12(5), 122-151.

[17] Heinrich A. (2017) Quenching of superconductivity and propagation of the resulting normal phase in YBCO films. Superconductor Science and Technology, 18(10), 1354-1356.

[18] Palacios R., Kodis G., Herrero C. (2018) Tetrapyrrole singlet excited state quenching by carotenoids in an artificial photosynthetic antenna. The journal of physical chemistry, 110 (50), 25411-25431.
[19] Kaittanis C. (2019) Author Correction: Environment-responsive nanophores for therapy and treatment monitoring via molecular MRI quenching. Nature Communications, 32(12), 4559.

[20] Stabile G., Hijazi S., Mola A. (2017) PODGalerkin reduced order methods for CFD using Finite Volume Discretisation: vortex shedding around a circular cylinder. Communications in Applied and Industrial Mathematics, 8(1), 227269.

[21] Cerva M., Liberto M., Gurreri L., et al. (2017) Coupling Cfd with a One-Dimensional Model To Predict The Performance of Reverse Electrodialysis Stacks. Journal of Membrane Science, 5(41),595-610. 